\title{
Measurements of the contact angle of Noa81 photoresist for different temperatures
}

\author{
Ekaterina O. Kirichenko, ${ }^{1,2,}$, and Elizaveta Ya. Gatapova ${ }^{1}$ \\ ${ }^{1}$ Kutateladze Institute of Thermophysics, 630090 Novosibirsk, Russia \\ ${ }^{2}$ Novosibirsk State University, 630090 Novosibirsk, Russia
}

\begin{abstract}
In this work we study the properties of the surface, obtained by applying Noa 81 photoresist at different temperatures of the substrate. The measurement of free energy was conducted by the Owens, Wendt, Rabel and Kaelble method under isothermal conditions. The paper presents the obtained data on contact angle for different temperatures.
\end{abstract}

\section{Introduction}

Intensive development of electronic technology is one of the main reasons for the continuous improvement of the existing cooling systems as well as designing brand new heat exchangers. This needs availability of advanced surfaces capable to resist thermal and mechanical stress [1]. Evaluating the possibility of using such surfaces in various devices, as well as studying physical phenomena near the three phases contact line $[2,3]$ plays a crucial role in designing advanced cooling systems.

This article examines the surface obtained by applying the Noa 81 photoresist. Using the Drop Shape Analyzer-E-100 produced by KRUSS, we investigated surface properties such as surface free energy and contact angle. By applying add-on TC40 module, which is a chamber for rapid change of temperature, the contact angle was measured within the substrate temperature range from 13 to $88^{\circ} \mathrm{C}$.

\section{Experimental setup and technique}

Studies of the contact angle and surface free energy were performed with the use of DSA 100 instrument produced by KRUSS. This device consists of three systems: high-precision dosing system with the dosing step of $0.1 \mu \mathrm{l}$, motorized object table for sample with program driven axes, and the optical system, which includes $50 \mathrm{~W}$ light source and a CCD camera with a resolution of $780 \times 580$ pixels (viewing field from $3.7 \times 2.7 \mathrm{~mm}$ to $23.2 \times 17.2$ $\mathrm{mm})$.

Free surface energy was measured using Owens, Wendt, Rabel and Kaelble method. Measurement technique is as follows. A few drops of water and diiodomethane are deposited on the surface. The surface tension is considered from the viewpoint of polar and disperse components. The method is based on the equation for the calculation of surface

* Corresponding author: kirichenko_ekaterina@,outlook.com 
tension combined with Young equation. The system is solved based on contact angles of two liquids with known polar and disperse components of the surface tension. Dispersion component includes van der Waals forces and other non-specific interactions, while polar component is responsible for strong interaction and hydrogen bonds. These considerations laid basis for derived equation:

$$
\frac{\sigma_{L}(\cos \theta+1)}{2 \sqrt{\sigma_{L}^{D}}}=\frac{\sqrt{\sigma_{S}^{P}} \cdot \sqrt{\sigma_{L}^{P}}}{\sqrt{\sigma_{L}^{D}}}+\sqrt{\sigma_{S}^{D}}
$$

The contact angle is investigated by forming a drop with a volume of $5 \mu$ ldeposited on the surface. The drop is formed by means of the syringe pump. Measurement of contact angle on the Noa 81 surface is shown in Fig. 1.

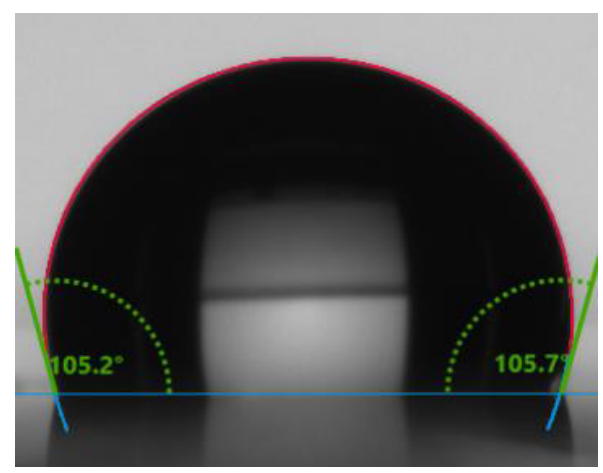

Fig. 1. Measurement of the contact angle on the Noa 81 surface.

In this article, the measurements were conducted within the temperature range from 13 to $88^{\circ} \mathrm{C}$ with the application of TC40 supplementary module with Peltier elements (Fig. 2).

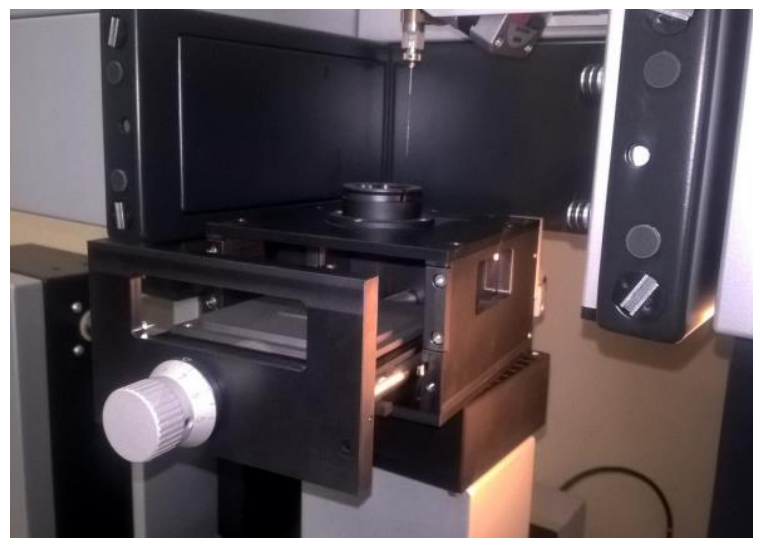

Fig. 2. The TC40 thermostatic chamber with Peltier elements.

The substrate is placed into a thermostatic chamber. A thermocouple is installed at a distance of $1 \mathrm{~mm}$ from the investigated surface to control ambient temperature. The liquid is fed through the hole from the top by means of the syringe pump. 


\section{Results}

The free energy of the Noa 81 surface substrate amounts to $23.24 \pm 0.66 \mathrm{mN} / \mathrm{m}$, at that, a dispersive component is equal to $22.83 \pm 0.46 \mathrm{mN} / \mathrm{m}$, while polar component is equal to $0.42 \pm 0.19 \mathrm{mN} / \mathrm{m}$. For example, for pure Teflon this value amounts to $18 \mathrm{mN} / \mathrm{m}$ while the polar component is missing.

Fig. 3 shows the dependence of contact angle on the surface temperature. Studies performed in a thermostatic chamber revealed that with increasing temperature the contact angle varies within the hysteresis. The contact angle hysteresis under isothermal conditions on a given surface was investigated in [4]. At an ambient substrate temperature, the contact angle hysteresis was $20^{\circ}$.

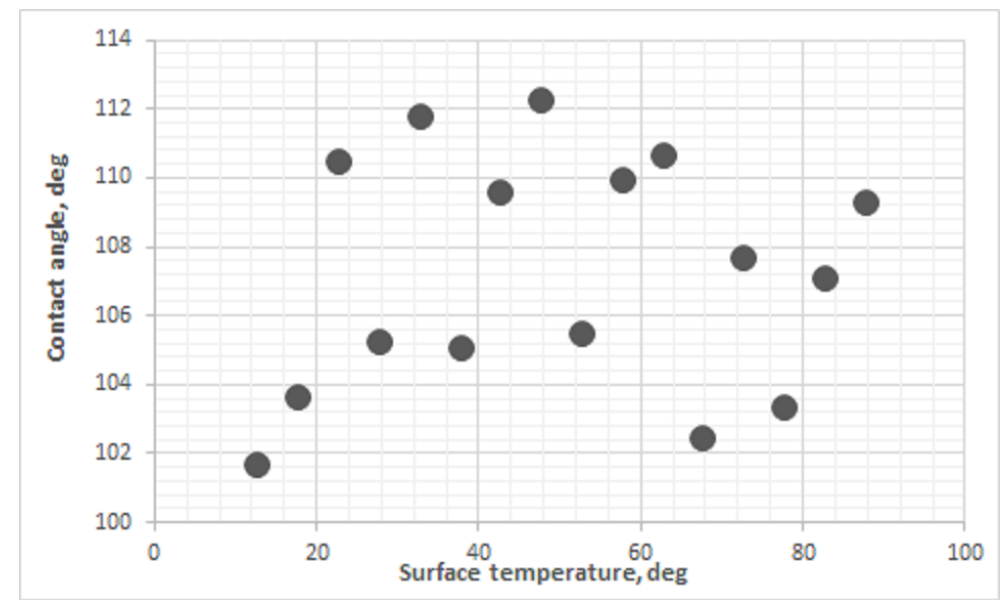

Fig. 3. The contact angle versus the surface temperature.

\section{Conclusions}

We have studied basic properties of the surface, obtained by applying the Noa 81 photoresist, carrying out measurements of a free surface energy and contact angle at different surface temperatures. It is shown that the contact angle varies within the hysteresis when changing surface temperature.

The authors gratefully acknowledge the support of this work by the Ministry of Education and Science of the Russian Federation (Agreement 14.616.21.0016, project identifier RFMEFI61614X0016).

\section{References}

1. S.R. Rao, Y. Peles, Int. J. Heat Mass Tran. 89, 482 (2015)

2. E.Y. Gatapova, O.A. Kabov, Microgravity Sci. Technol. 19, 132 (2007)

3. A. Zou, A. Chanana, A. Agrawal, P.C. Wayner Jr., S.C. Maroo1 Sci. Rep. 6, 20240 (2016)

4. E.O. Kirichenko, E.Y. Gatapova, J. Phys. Conf. Ser. 754, 032009 (2016) 